\title{
Estimation of Delivered Dose in Radiotherapy: The Influence of Registration Uncertainty
}

\author{
Petter Risholm ${ }^{1}$, James Balter ${ }^{2}$, and William M. Wells ${ }^{1}$ \\ 1 Brigham and Women's Hospital, Harvard Medical School \\ 2 Department of Radiation Oncology, University of Michigan \\ pettri@bwh.harvard.edu
}

\begin{abstract}
We present a probabilistic framework to estimate the accumulated radiation dose and the corresponding dose uncertainty that is delivered to important anatomical structures, e.g. the primary tumor and healthy surrounding organs, during radiotherapy. The dose uncertainty we report is a direct result of uncertainties in the estimates of the deformation which aligns the daily cone-beam CT images with the planning $\mathrm{CT}$. The accumulated radiation dose is an important measure to monitor during treatment, in particular to see if it significantly deviates from the planned dose which might indicate that either the patient was not properly positioned before treatment or that the anatomy has changed due to the treatment. In the case of the latter, the treatment plan should be adaptively changed to align with the current patient anatomy. We estimate the accumulated dose distribution, and its uncertainty, retrospectively on a dataset acquired during treatment of cancer in the neck and show the dose distributions in the form of dose volume histograms.
\end{abstract}

\section{Introduction}

Oral and pharyngeal cancers are a significant cause of morbidity and mortality. Approximately 36000 Americans will be diagnosed with such cancers in 2011, and, of these, only half will survive more than 5 years. Radiotherapy (RT) for cancer in the neck involves extreme precision in planning, image guidance, and delivery, due to the complex distribution of possible tumor locations and their close proximity to a large variety of healthy organs. Using intensity modulated radiation therapy (IMRT), highly sculpted dose distributions can be planned for achieving the dual goals of treating the primary tumor and nearby tissue suspected of containing disease with high doses, while limiting the dose, and thus toxicity risk, to healthy organs.

RT of cancer in the neck region is commonly delivered in 30-35 fractions over 6-7 weeks where the patient must be positioned appropriately for each fraction in order to receive the treatment dose [7. On any day, the position, orientation, and configuration may vary due to the random nature of the immobilization and setup processes [3]. In addition, chronic changes in response to the treatment and its side effects will manifest as shape changes in the tumor, which typically diminishes in size as treatment progresses, and other tissue changes in the neck

G. Fichtinger, A. Martel, and T. Peters (Eds.): MICCAI 2011, Part I, LNCS 6891, pp. 548 555, 2011. (C) Springer-Verlag Berlin Heidelberg 2011 
due to e.g. weight loss. The typical method employed for ensuring that the dose is delivered safely and effectively on a given day involves changing the position of the patient under image guidance. Such changes, however, are typically restricted to simple translational adjustments 8], as most linear accelerators are not equipped with rotational adjustment systems.

To understand the deviation of dose delivered to a patient from that planned, and thus to guide decisions to adaptively modify treatment plans, it is necessary to accumulate the dose delivered to an important tissue structure as treatment progresses. By non-rigidly aligning the daily Cone-Beam Computed Tomography (CBCT) image with the Computed Tomography (CT) acquired for planning [4], the dose can be summed up for various tissues as treatment progresses and is commonly presented in the form of Dose Volume Histograms (DVH) 1 .

The large variety of non-rigid registration methods available have different possible biases as well as random variations due to their driving algorithms and the signals (or degeneracy of signals) available to drive them to optimal solutions. Most methods are deterministic and disregard any concept of uncertainties which may manifest in inaccurate contour mapping and dose reporting. This may lead to erroneous conclusions when deciding whether certain shape changes and their resulting dosimetric impacts warrant more significant intervention than patient position adjustment. In this work we propose a probabilistic framework for estimating the accumulated radiation dose delivered during IMRT in the neck. The daily CBCT is non-rigidly registered with the planning CT using a probabilistic registration framework [56] that quantifies the deformation as well as the deformation uncertainty. We show how the delivered dose estimates are influenced by registration uncertainty.

\section{Methods}

Based on the planning CT image $\mathbf{c}(\mathbf{x})$ and the corresponding binary images of critical structures (e.g. spinal cord, tumor, etc) $\mathbf{s}_{1}(\mathbf{x}), \ldots, \mathbf{s}_{K}(\mathbf{x})$, a radiation dose volume $\mathbf{r}(\mathbf{x})$ is determined, where $\mathbf{x} \in \Omega$ and $\Omega$ denotes the treatment domain. The total dose is delivered over $T$ identical dose fractions $\mathbf{r}_{1}=\ldots=\mathbf{r}_{T}$ such that $\mathbf{r}=\mathbf{r}_{1}+\ldots+\mathbf{r}_{T}$. Before each fraction, a new CBCT image $\mathbf{f}_{t}$ is acquired and used to find a translational (rigid) alignment of the patient with respect to the pre-treatment plan.

However, a rigid alignment cannot account for non-rigid anatomical changes, and thus, in those places where non-rigid shifts occur, the dose will not be delivered accurately to the planned treatment site. By estimating the non-rigid deformation $\mathbf{u}_{t}$ which aligns $\mathbf{f}_{t}$ with $\mathbf{c}$, we can estimate the deviation between the planned radiation and the actual radiation $\mathbf{d}_{t}$ delivered to a structure $\mathbf{s}_{i}$ at fraction $t$. We model $p\left(\mathbf{d}_{T}^{*}\right)$, the accumulated marginal dose distribution after fraction $T$, according to the hierarchical model in Fig. 1](and drop the conditional dependence of $\mathbf{d}$ on $\mathbf{r}, \mathbf{c}$ and $\mathbf{f}$ for notational convenience): 


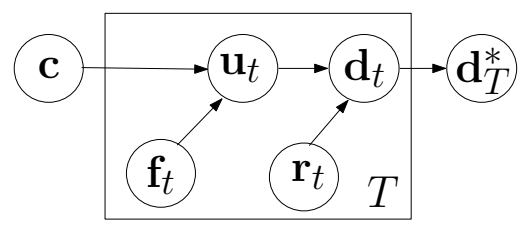

Fig. 1. Hierarchical model of the problem of estimating the actual accumulated radiation dose $\mathbf{d}_{T}^{*}$ after $T$ fractions given the planning CT $\mathbf{c}$, the daily CBCT images $\mathbf{f}_{t}$ and the planned per-fraction dose images $\mathbf{r}_{t}$. The deformations $\mathbf{u}_{t}$ and the actual per-fraction doses $\mathbf{d}_{t}$ are unknowns.

$$
\begin{array}{r}
p\left(\mathbf{d}_{T}^{*}\right)=p\left(\mathbf{d}_{1}\right) * \ldots * p\left(\mathbf{d}_{T}\right), \\
p\left(\mathbf{d}_{t}\right)=\int_{\mathbf{u}_{t}} p\left(\mathbf{d}_{t} \mid \mathbf{u}_{t}, \mathbf{r}_{t}\right) p\left(\mathbf{u}_{t} \mid \mathbf{c}, \mathbf{f}_{t}\right),
\end{array}
$$

where we can estimate $p\left(\mathbf{d}_{T}^{*}\right)$ by convolution of $p\left(\mathbf{d}_{t}\right)$ for $t=1, \ldots, T$ because they are conditionally independent given $\mathbf{r}_{t}$, $\mathbf{c}$ and $\mathbf{f}_{t}$. In the following sections we show how to compute the posterior distribution over deformations, the conditional dose distribution, the marginal per-fraction dose distribution, and finally the accumulated dose distribution.

\subsection{Probabilistic Non-rigid Registration}

Here we provide a summary of the probabilistic registration framework in $[56$ where the posterior distribution on deformations is written

$$
p\left(\mathbf{u}_{t} \mid \mathbf{c}, \mathbf{f}_{t}\right) \propto p\left(\mathbf{c}, \mathbf{f}_{t} \mid \mathbf{u}_{t}\right) p\left(\mathbf{u}_{t}\right) .
$$

In the deformation likelihood $p\left(\mathbf{c}, \mathbf{f}_{t} \mid \mathbf{u}_{t}\right)=\frac{1}{Z_{s}} \exp \left(-E_{s} / T_{s}\right)$ we convert a Sum of Squared Differences (SSD) similarity criterion $E_{s}\left(\mathbf{u}_{t} ; \mathbf{c}, \mathbf{f}_{t}\right)$ into a probability by way of Boltzmann's equation with temperature $T_{s}$. Similarly in the prior $p\left(\mathbf{u}_{t}\right)=\frac{1}{Z_{e}} \exp \left(-E_{e} / T_{e}\right)$, Boltzmann's equation with temperature $T_{e}$ is used to convert a non-linear elastic (St. Venant-Kirchoff) energy $E_{e}\left(\mathbf{u}_{t}\right)$ into a probability. The computation domain is discretized with a Finite Element (FE) tetrahedral mesh which is used to calculate both $E_{s}$ and $E_{e}$ and we prevent folding of elements by setting the elastic energy to infinity when folding is detected.

The method uses Metropolis-Hastings (MH), a Markov Chain Monte Carlo (MCMC) method, to draw deformation samples from the posterior distribution 2]. The MH method constructs a Markov chain that asymptotically has the posterior distribution as its equilibrium distribution. In practice, the method starts with an initial estimate of the deformation $\mathbf{u}^{0}$, and generates candidate samples from a multi-variate Gaussian proposal distribution centered on the previous sample $\mathbf{u}^{*} \sim \mathcal{N}\left(\mathbf{u}^{t}, \sigma^{2} \boldsymbol{\Sigma}\right)$. The covariance matrix is defined by $\Sigma_{i j}=$ $\exp \left(-\mathcal{D}\left(n_{i}, n_{j}\right) / \rho\right)$ where $\mathcal{D}$ computes the distance between nodes $i$ and $j$ in 
the FE-mesh and $\rho$ is a constant. Candidate samples are accepted $\left(\mathbf{u}^{t+1}=\mathbf{u}^{*}\right)$, or rejected $\left(\mathbf{u}^{t+1}=\mathbf{u}^{t}\right)$, according to the $\mathrm{MH}$ criterion [2]. Typically, the first set of samples is discarded to avoid burn-in effects, and to reduce intra-sample dependencies the chain is often thinned by saving every $k$-th sample. Eventually, a set of deformations $\hat{\mathbf{u}}=\left\{\mathbf{u}^{i}\right\}_{i=1 \ldots N}$ that represent the posterior distribution is generated.

\subsection{Marginal Radiation Dose Distributions}

We assume dose invariance with respect to anatomical changes and therefore model the conditional per-fraction dose distribution $p\left(\mathbf{d}_{t} \mid \mathbf{u}_{t}, \mathbf{r}_{t}\right)=\delta\left(\mathbf{d}_{t}-\mathbf{r}_{t}\right.$ 。 $\mathbf{u}_{t}$ ) with a delta distribution. Consequently, we characterize the marginal dose distribution in Eq. (2) by the set of deformed dose samples $\hat{\mathbf{d}}_{t}=\left\{\mathbf{r}_{t} \circ \mathbf{u}^{i}\right\}_{i=1 \ldots N}$ where $\circ$ denotes composition.

We are interested in monitoring the accumulated dose delivered to a structure $\mathbf{s}_{i}$ over the duration of the treatment. A simple way to estimate $p\left(\mathbf{d}_{T}^{*}\right)$, given that we have samples that characterize the per-fraction marginal dose distributions, is by drawing a single random sample from each of the per-fraction dose distributions and adding them together. We draw a set of $M=T N k$ samples $\hat{\mathbf{d}}_{T}^{*}$ to characterize $p\left(\mathbf{d}_{T}^{*}\right)$, where $k$ is a constant that determines the amount of over-sampling we need to get good coverage of the distribution.

A DVH summarizes a 3D dose distribution for a specific structure $\mathbf{s}_{i}$ in a graphical 2D format and is used for both planning and monitoring radiation treatment [1. We use it to visualize the most likely radiation dose delivered to a specific structure, either at a single fraction, or the cumulative dose over several fractions. In addition to visualizing the most likely dose distribution, we also visualize the uncertainty (spread) of the dose distribution by plotting the DVHs corresponding to the minimum and maximum limit of the range of the accumulated dose distribution represented by the samples in $\hat{\mathbf{d}}_{T}^{*}$.

\subsection{Marginal Contour Distributions}

It is difficult to assess the final registration results because of the lack of ground truth. We propose to assess the registration through $p\left(\mathbf{s}_{j} \circ \mathbf{u}^{-1}\right)$, i.e. the marginal posterior location of the critical structures in the planning image to see if they align with the structure seen in the daily CBCT. It is especially critical to compare the estimated location of these structures with the planned location and the planned dose distribution for those structures where the estimated DVH starts diverging from the planned DVH. A marginal posterior map of the location of the structure in the CBCT can be constructed by deforming the structure with the inverse of each of the sampled deformations $\left\{\mathbf{s}_{j} \circ\left(\mathbf{u}^{i}\right)^{-1}\right\}_{i=1 \ldots N}$ and making a histogram of the number of times a voxel is within the structure. We can view this $3 \mathrm{D}$ histogram as a probabilistic map which conveys the probability that a voxel belongs to the structure given the posterior distribution over deformations. 

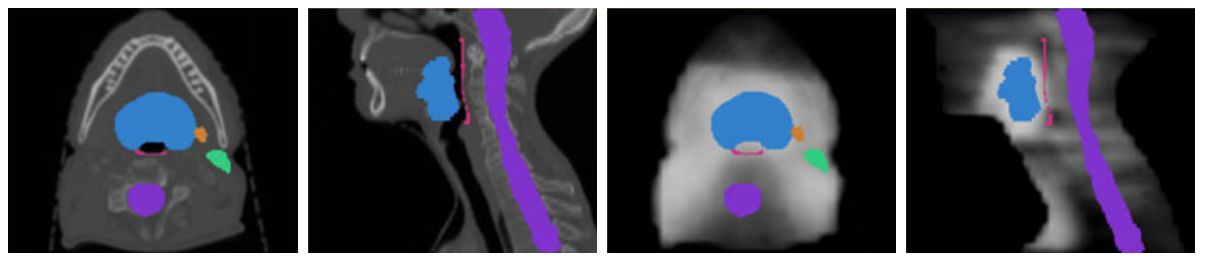

Fig. 2. RT planning data. In the two leftmost images, critical structures (Blue=PTV70, Red $=$ Pharyngeal constrictors, Orange $=$ Left submandibular gland, Green=Left parotid gland, Purple=Spinal cord) used in planning of the radiation dose are overlaid on axial and sagittal slices of the planning CT. In the two rightmost images, corresponding structures are overlaid on axial and sagittal slices of the planned dose $\mathbf{r}$. Notice the steep dose gradients between the spinal cord, pharyngeal constrictor and PTV70.

\section{Results}

Dataset. We applied our method retrospectively to a Head \& Neck data set which consisted of a planning CT $(512 \times 512 \times 135)$ with resolution $(0.95,0.95,3.00)$ mm. Important critical structures were delineated on this CT image (see Fig. 2) and a dose volume $(61 \times 47 \times 45)$ with isotropic $5 \mathrm{~mm}$ resolution was estimated based on the contoured data to maximize the tumor radiation and minimize the radiation to healthy tissue. In the rest of this paper, we will monitor DVHs of normal structures pharyngeal constrictor, brain stem, left submandibular gland and left parotid gland to confirm that the actual dose delivered does not exceed the planned dose, and the planning target volume (PTV70) to monitor that the delivered dose does not fall below the planned dose.

The patient came in for 35 fractions over 7 weeks, and at each fraction a CBCT $(512 \times 512 \times 61)$ with a resolution of $(0.5,0.5,2.5) \mathrm{mm}$ was acquired to find a translational alignment of the patient with the treatment scan. We used the CBCT from end of week 1, 4 and 7 and assumed that 1/3 of the total dose was applied at each of these time points. We registered the CBCTs to the planning CT to monitor the cumulative DVH for each structure and how they are affected by registration uncertainty and tissue movements due to changing anatomy.

Registration. First we estimated a translation which best aligned the CBCT with the planning CT and assumed this reproduced the translational alignment used during treatment. We generated a FE-mesh, with 1402 nodes and 6398 tetrahedral elements, which covered the treatment region of the neck and used homogeneous elastic parameters with Young's modulus and Poisson's ratio set to $E=10^{8}$ and $\nu=0.45$ respectively. Next we applied our probabilistic registration $\left(\sigma^{2}=0.008, \rho=50 \mathrm{~mm}, T_{e}=10^{8}, T_{s}=1.0\right)$, sampled 400000 samples, used a thinning-factor of 10 , and removed the first half of the data to avoid burnin effects. Effectively, we had 20000 independent samples from the posterior 

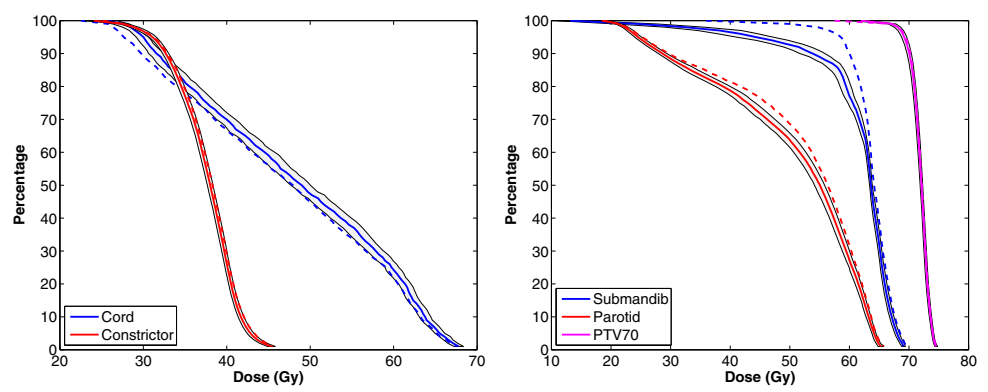

Fig. 3. Cumulative DVH after week 7 for the spinal cord and pharyngeal constrictor on the left and submandibular gland, parotid gland and PTV70 on the right. The gray area is the uncertainty of the DVH and the dashed line is the planned dose. Notice the large deviation from the plan for the submandibular gland and that the planned DVH for the parotid gland falls outside of the estimated DVH. The large deviation from the plan indicates anatomical changes and that the treatment can be replanned. Fig. 4 plots the DVHs for the parotid and submandibular gland from week 1 to 7 .

distribution for each dataset. The temperatures were set empirically to balance the contribution of the likelihood and the prior, while $\sigma^{2}$ was set to achieve an $\mathrm{MH}$ acceptance rate of approximately $25 \%$.

Dose Estimation. From the per-fraction dose distributions we estimated the cumulative dose distribution per voxel with $k=100$. In Fig. 3 we show the cumulative DVHs after week 7 and the corresponding uncertainty (dispersion) of the estimates for the 5 different structures we are studying. It is apparent from Fig. 3 that there are deviations in the estimated DVH compared to the planned DVH for the submandibular and parotid glands. In Fig. 4 we study the accumulated dose for these structures closer and plot the cumulative DVH for weeks 1, 4, and 7. After week 1 , the actual radiation delivered to the submandibular gland apparently diverges from the planned radiation and is an indication that the tissue in or around the left submandibular gland has moved/changed during treatment and that large dose gradients close to the structure have moved in or out of the structure.

In Fig. [5 we visualize the marginal position of the submandibular and parotid glands overlaid on the week $7 \mathrm{CBCT}$ based on the estimated posterior distribution over deformations to 1) visually validate the registration and 2) to assess if the tissue has moved/changed such that the structure contoured in the treatment CT no longer overlaps with the marginal location of the contour. For the submandibular gland, it is evident that the tissue has moved such that large dose gradients that were previously outside the structure are now inside, which means that the structure is receiving a lower amount than the planned dosage. This might be an indication to re-plan the treatment and possibly taking further advantage of the possible dose sparing to this organ. 

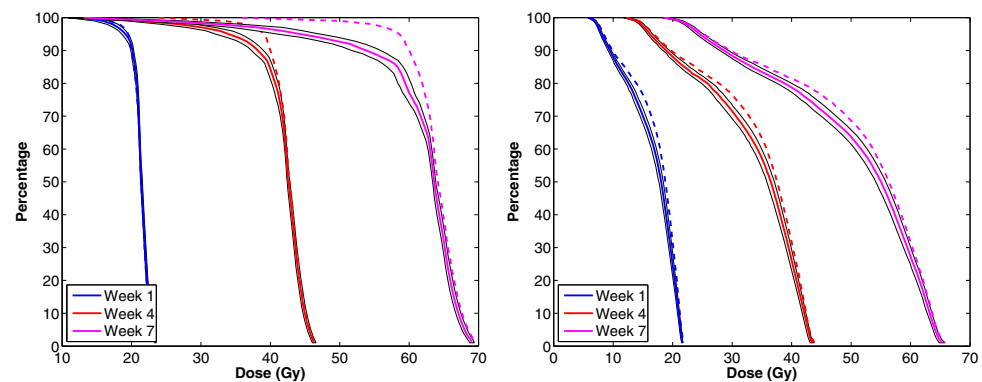

Fig. 4. Cumulative DVHs for the submandibular (left) and the parotid (right) glands. The dashed line is the planned dose, while the gray area is the dose uncertainty. Because we see a large uncertainty in the cumulative DVH for the parotid and submandibular glands in Fig. 3] we plot here the cumulative DVH after weeks 1, 4 and 7 to study the temporal evolution of the delivered dose distributions. At week 4, the estimated DVH for the submandibular gland starts to diverge from the planned DVH. To confirm that these deviations are due to changing and moving anatomy, we plot the marginal distribution of the glands in relation to the planned position in Fig. [5

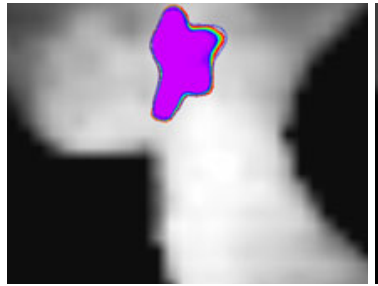

(a) $p\left(\mathbf{s}_{\text {parot }} \circ \mathbf{u}_{7}^{-1}\right)$ and $\mathbf{s}_{\text {parot }}$

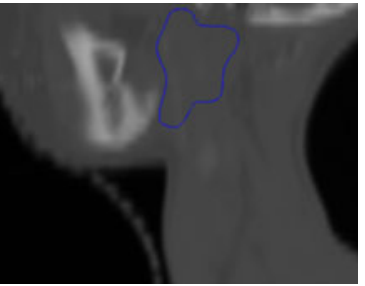

(b) $\mathbf{s}_{\text {parot }}$

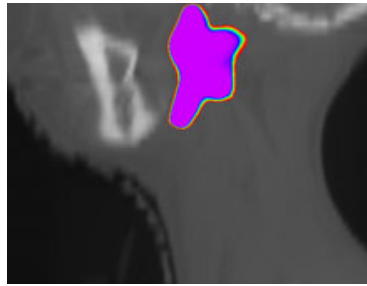

(c) $p\left(\mathbf{s}_{\text {parot }} \circ \mathbf{u}_{7}^{-1}\right)$

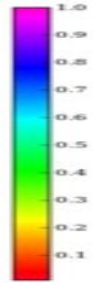

(d)

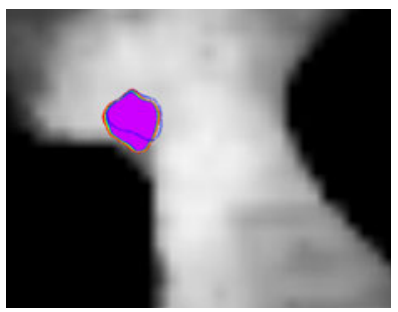

(e) $p\left(\mathbf{s}_{\mathrm{subm}} \circ \mathbf{u}_{7}^{-1}\right)$ and $\mathbf{s}_{\mathrm{subm}}$

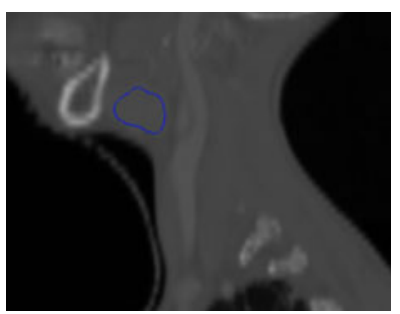

(f) $\mathbf{S}_{\text {subm }}$

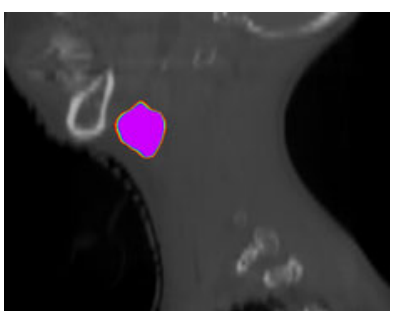

(g) $p\left(\mathbf{s}_{\text {subm }} \circ \mathbf{u}_{7}^{-1}\right)$

Fig. 5. Marginal maps and planned contours of the parotid and submandibular glands. The probability of a voxel being inside the marginal contour is colored according to $(d)$. (a, e): Marginal maps and planned location (in blue) overlaid on $\mathbf{r}$. Notice the deviation of the real location of the submandibular gland (according to the marginal map) from the planned location and how this translates into a deviation in the DVHs (see Fig. 4) because of the large dose gradients in this area. (b, f): Planned contours overlaid on c. (c, g): Marginal maps overlaid on $\mathbf{f}_{7}$ (week $7 \mathrm{CBCT}$ ). Notice the uncertainty in the location of the upper right part of the parotid gland. However, this locational uncertainty does not translate into a large uncertainty in the DVH (see Fig. 3) because there are no large dose gradients in this area. 


\section{Discussion}

This paper presented a probabilistic framework for estimating the cumulative radiation dose delivered to tissue during IMRT and the corresponding dose uncertainty contributed by non-rigid registration. Our preliminary results show that the dose uncertainty varies with the structure we are studying and whether it is close to large dose gradients. The resolution of the dose volume was low which resulted in relatively small dose uncertainties. With finer resolution dose volumes and by accumulating dose from more than three fractions, we anticipate that the dose uncertainties will play a role in deciding whether to re-plan the treatment. The uncertainty estimates also depend on the Boltzmann temperatures. In future work, we will use a likelihood approach to estimate physical meaningful temperatures, and we will also differentiate between tissue types by modeling them with different elasticity. Our current similarity metric (SSD) is not a robust measure, but was used for its computational efficiency. MCMC sampling is generally slow, and we achieved a sampling frequency of 10 samples per second on a 8-core PC for a total computation time of about $11 \mathrm{~h}$ for one fraction. However, these computations can be done offline between fractions and computational speed is therefore not critical.

Acknowledgments. The work was supported by NIH grants P01CA59827, P41RR13218 and P41RR019703.

\section{References}

1. Drzymala, R., Mohan, R., Brewster, L., Chu, J., Goitein, M., Harms, W., Urie, M.: Dose-volume histograms. Int. J. Radiat. Oncol. Biol. Phys. 21(1), 71-78 (1991)

2. Gelman, A., Carlin, J.B., Stern, H.S., Rubin, D.B.: Bayesian Data Analysis, 2nd edn. Chapman \& Hall/CRC (July 2003)

3. Hong, T.S., Tome, W.A., Chappell, R.J., Chinnaiyan, P., Mehta, M.P., Harari, P.M.: The impact of daily setup variations on head-and-neck intensity-modulated radiation therapy. Int. J. Radiat. Oncol. Biol. Phys. 61(3), 779-788 (2005)

4. Lu, W., Olivera, G.H., Chen, Q., Ruchala, K.J., Haimerl, J., Meeks, S.L., Langen, K.M., Kupelian, P.A.: Deformable registration of the planning image (kvct) and the daily images (mvct) for adaptive radiation therapy. Phys. Med. Biol. 51(17), 4357 (2006)

5. Risholm, P., Pieper, S., Samset, E., Wells III, W.M.: Summarizing and visualizing uncertainty in non-rigid registration. In: Jiang, T., Navab, N., Pluim, J.P.W., Viergever, M.A. (eds.) MICCAI 2010. LNCS, vol. 6362, pp. 554-561. Springer, Heidelberg (2010)

6. Risholm, P., Samset, E., Wells III, W.: Bayesian estimation of deformation and elastic parameters in non-rigid registration. In: Fischer, B., Dawant, B.M., Lorenz, C. (eds.) WBIR 2010. LNCS, vol. 6204, pp. 104-115. Springer, Heidelberg (2010)

7. Schwartz, D.L., Dong, L.: Adaptive radiation therapy for head and neck cancer-can an old goal evolve into a new standard? J. Oncol. (2011)

8. Sykes, J.R., Brettle, D.S., Magee, D.R., Thwaites, D.I.: Investigation of uncertainties in image registration of cone beam ct to ct on an image-guided radiotherapy system. Phys. Med. and Biol. 54(24), 7263 (2009) 